\title{
Comparing cost-effectiveness of standardised tuberculosis treatments given varying drug resistance
}

\author{
Stephanie Law, Andrea Benedetti, Olivia Oxlade, Kevin Schwartzman and \\ Dick Menzies
}

Affiliation:

Respiratory Epidemiology and Clinical Research Unit, McGill University, Montreal, Canada.

Correspondence:

D. Menzies, Room K1.24, Montreal Chest Institute, 3650 St Urbain St, Montreal, H2X 2P4, PQ, Canada.

E-mail: Dick.MenziesaMcGill.ca

ABSTRACT There is a growing need to identify appropriate standardised treatment strategies that will adequately treat various forms of drug-resistant tuberculosis (TB) and prevent multidrug-resistant (MDR)-TB.

A Markov model estimated treatment-related acquired MDR-TB, mortality, disability-adjusted life years and costs in settings with different prevalence of isoniazid monoresistant TB and MDR-TB. We compared four treatment strategies: 1) the standard World Health Organization recommended treatment strategy; 2) adding ethambutol throughout the 6-month treatment of new cases; 3) using a strengthened standardised retreatment regimen; and 4) using standardised MDR treatment for failures of initial treatment. Treatment-related outcomes were derived from the published literature, and costs from direct surveys.

A strengthened retreatment regimen, which could achieve lower failure, relapse and acquired MDR rates in isoniazid monoresistant cases, was predicted to be the most cost-effective strategy in all modelled settings. Empirical MDR treatment of failures of initial treatment was the most costly strategy but resulted in the fewest deaths. Adding ethambutol throughout initial treatment would be most effective in preventing acquired MDR, but would lead to excess cases of blindness.

A high priority should be given to improving the standardised retreatment regimen, as this is predicted to produce greater benefits than other recently recommended strategies.

@ERSpublications

Priority should be given to improving the standardised TB retreatment regimen http://ow.ly/r54us

This article has supplementary material available from www.erj.ersjournals.com

Received: Jan 102013 | Accepted after revision: June 072013 | First published online: June 132013

Support statement: Funding for this study was provided by the Canadian Institutes of Health Research (CIHR Frederick Banting and Charles Best Canada Graduate Scholarships - Master's Award).

Conflict of interest: Disclosures can be found alongside the online version of this article at www.erj.ersjournals.com

Copyright @ERS 2014 


\section{Introduction}

The World Health Organization (WHO) estimated that in 2011 there were 8.7 million new tuberculosis (TB) cases and 1.4 million TB deaths [1]. As a consequence of inadequate TB treatment and management, drug-resistant TB has emerged in all parts of the world and now accounts for $\sim 17 \%$ of all new cases [2]. The most common form of drug-resistant TB is isoniazid (INH) resistance, which is seen in $10 \%$ of new cases [2]. INH resistance is of concern as INH is one of the two most effective and potent first-line anti-TB drugs, and approximately one-third of new cases with INH resistance, or 3\% of all new cases, has multidrug-resistant (MDR)-TB, which is a strain resistant to both INH and rifampicin (RMP) [2]. Patients with MDR-TB have substantially worse treatment outcomes [3,4], despite a course of treatment that is much longer [5], and is more toxic and expensive [1], than for drug-sensitive TB. The proportions of MDR-TB have been increasing in many parts of the world; the highest proportions recorded in the history of the WHO Global Project on Anti-tuberculosis Drug Resistance Surveillance reached 35.3\% among new and $76.5 \%$ of previously treated TB cases in 2012 in Minsk, Belarus [6].

Globally, $<4 \%$ of all new cases of TB are estimated to receive drug susceptibility testing [1]. Thus, the vast majority of drug-resistant TB patients are unrecognised and do not receive adequate treatment [2]. In order to prevent MDR-TB where proper diagnostic testing is unavailable, the WHO recommends empirically adding ethambutol (EMB) throughout the initial treatment of new cases in settings with high levels of INH resistance [7]. However, the ability of EMB to protect against MDR-TB is uncertain [8, 9], and should be weighed against the risk of ocular toxicity [10-14]. The WHO also recommends using an 8-month regimen for previously treated patients who relapsed or defaulted [7]. This standardised retreatment regimen is comprised entirely of first-line drugs, was designed for populations with low levels of INH resistance and has never been tested in randomised clinical trials [4]. Published reports point towards unacceptably high rates of treatment failure, relapse and acquired drug resistance with this regimen in the presence of (nonMDR) INH resistance $[3,4]$. Given that $\sim 28 \%$ of all retreatment TB cases are resistant to INH globally [2], this regimen will probably be frequently unsuccessful [3]. A third related WHO recommendation is to use an empirical MDR regimen for patients who have failed initial treatment [15].

The effectiveness of these three WHO treatment strategies is unclear in the context of emerging drugresistant TB. In this study, we compared the treatment outcomes and costs of different strategies in four settings with different prevalence of INH monoresistance and/or MDR. The four strategies compared were: 1) the current WHO standard initial and retreatment guidelines; 2) adding EMB throughout initial treatment of new cases; 3) using a hypothetical retreatment regimen strengthened with second-line TB drugs; and 4) treating all failures of initial treatment with an empirical MDR regimen.

\section{Methods}

Overview

We estimated the treatment outcomes and costs of four different treatment strategies over a 10-year analytical horizon, using a Markov decision analysis model. We adhered to published recommendations for conducting and reporting a cost-effectiveness analysis [16, 17]. We assumed a societal perspective to evaluate costs and discounted both costs and health effects at a 3\% annual rate. Our model assumed that drug susceptibility testing was not available and did not influence the treatment given, thus all treatment regimens modelled were standardised. There is insufficient published literature for estimating input parameters specific to HIV-TB co-infected patients; thus, we assumed that patients with HIV co-infection would have similar treatment outcomes [18].

\section{Decision analytic model}

We used TreeAge Pro 2008 Suite (release 1.0; TreeAge Software, Inc., Williamstown, MA, USA) to construct the Markov model. The model described the clinical progression of TB disease (fig. 1). A 10-year analytical horizon was used for comparability with previously published cost-effectiveness studies examining TB treatment strategies $[19,20]$.

The model simulated the treatment outcomes and costs accrued over 10 years for four hypothetical cohorts of 100000 smear-positive, treatment-nä̈e TB patients. Each cohort went through the Markov model under four different treatment strategies (described later). When a cohort entered the model, its patient population was distributed across three initial health states, known as Markov states, which described patients with different primary drug resistance profiles (drug-susceptible TB, INH monoresistant TB and MDR-TB) undergoing initial treatment for TB. Only two forms of drug-resistant TB were modelled because there are insufficient published data regarding treatment outcomes of other forms of drug resistance. Patients with any resistance to RMP were considered to be in the same health state as MDR-TB patients 
Treatment strategies:

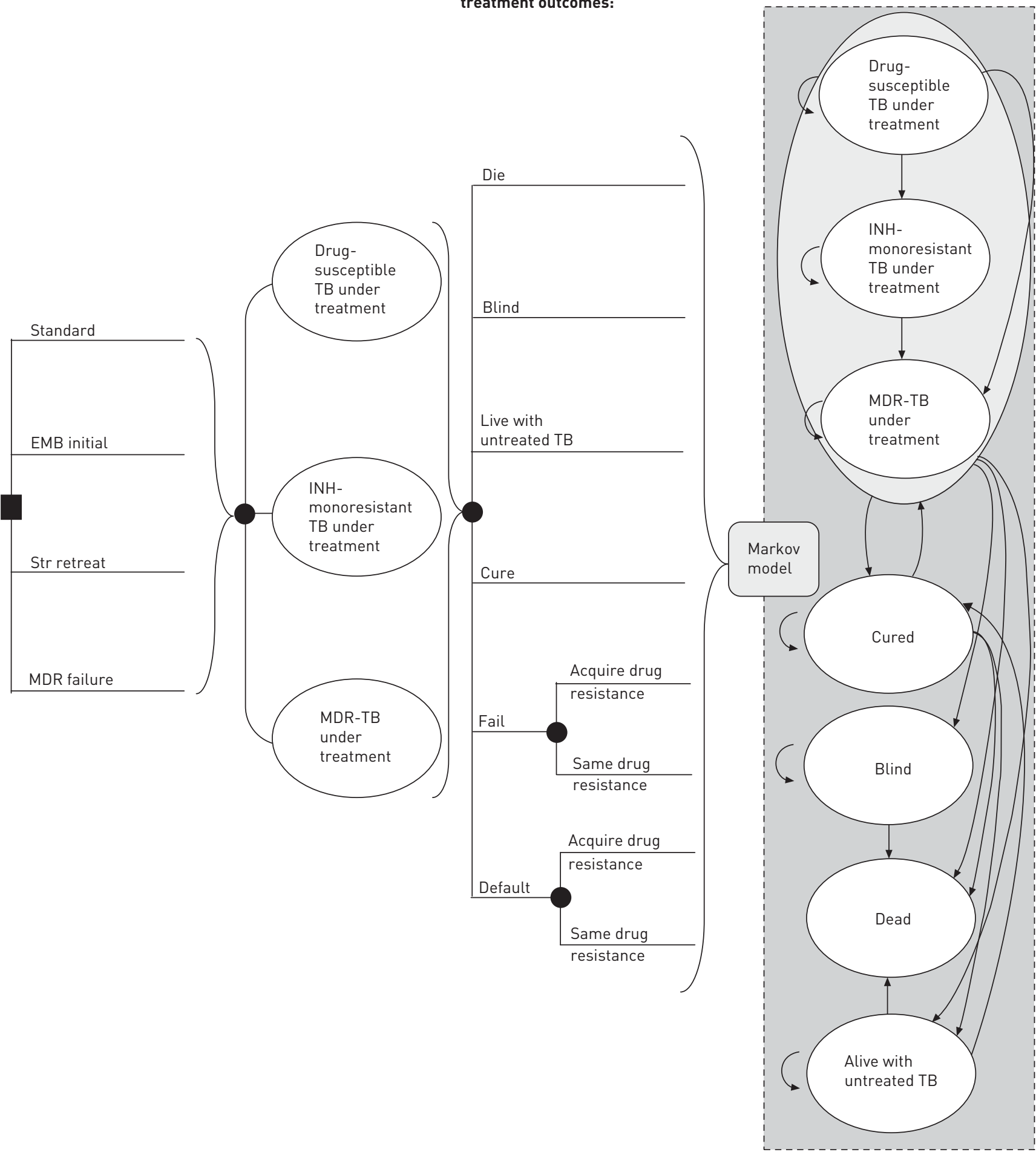

FIGURE 1 Schematic representation of the decision analytic model. The ovals represent the seven different Markov health states. The solid square on the left represents the initial decision regarding which treatment strategy to apply. The solid circles are probability nodes, meaning a pre-defined probability at that node determined the outcome thereafter. These probability nodes make up the probability tree that patients go through to determine their next Markov state after every cycle spent in an "under treatment" Markov state. Throughout the 10-year time horizon, patients transitioned between the Markov states based on transition probabilities. The Markov state "dead" is an absorbing state, which means once patients entered the dead state, they could not transition to other states. Patients entered the "alive with untreated [tuberculosis] TB" state only if they failed, defaulted or relapsed after receiving the standardised multidrug-resistant (MDR) regimen. If a patient entered the "cured" Markov state, they could relapse within the next 1-year cycle. EMB: ethambutol; Str retreat: strengthened retreatment; INH: isoniazid. 
because RMP resistance is considered a surrogate marker for MDR, and has significantly worse outcomes than other forms of drug resistance [21-26].

Each cohort represented a different setting with varying prevalence of primary INH monoresistance and MDR-TB in treatment-naïve patients, as follows: 1) low prevalence of both forms of drug resistance $(5 \%$ INH monoresistant and $1 \%$ MDR-TB); 2) high prevalence of INH monoresistance (15\%) and low prevalence of MDR-TB (1\%); 3 ) low prevalence of INH monoresistance (5\%) and high prevalence of MDRTB (10\%); and 4) high prevalence of both forms of drug resistance (15\% INH monoresistant and $10 \%$ MDR-TB).

The cycle length of the model was 1 year, which was chosen in order to accommodate the following different treatment lengths: 6 months for initial treatment regimens; 8 months for retreatment regimens; and 24 months for the MDR regimen, which took two cycles. After the first year in the model, patients all moved through a probability tree that determined the Markov state they would be in during the following year. Depending on transition probabilities, patients could fail, default or successfully complete treatment and be cured; become blind due to the ocular toxicity of ethambutol; or die from TB or non-TB causes. Those who failed or defaulted treatment could undergo treatment the following year; however, their underlying drug resistance could have changed and the new regimen they received depended on the treatment strategy being modelled. Those who successfully completed treatment would be in the "cured" state but could relapse and return to treatment; for simplicity, we assumed that all relapses would occur only in the year immediately following treatment completion. Estimates of transition probabilities and treatment outcomes were derived from published literature whenever possible and expert clinical judgement when not (table 1).

After each yearly cycle, patients either transitioned to a different Markov state or remained in the same state. How a patient progressed through the model depended on a patient's clinical history and underlying drug resistance profile.

\section{Probability of EMB-induced blindness}

If a patient became blind at any point during treatment, their treatment was discontinued in our model. This assumption was based on the understanding that blindness is detrimental to the livelihood of patients in resource-limited countries and, critically, curtails their ability to remain in treatment. The probability of becoming blind due to EMB was estimated at $0.23 \%$ (95\% CI $0-0.61 \%$ ) for a 6-month EMB treatment, based on results of a systematic review and meta-analysis [14].

\section{Model outcomes}

Each health state had corresponding health outcomes (table 1) and costs (online supplementary table S1). At the end of each year, the costs and health outcomes were accrued for the patients. Patients who completed treatment accrued the total cost of treatment. There were no costs assigned to the states "dead", "blind", "cured" and "alive with untreated TB".

Our model results were expressed in terms of costs, INH monoresistant cases prevented, MDR-TB cases prevented, excess blindness, TB-related deaths averted and disability-adjusted life years (DALYs) gained. DALYs are an effectiveness measure introduced by the WHO to combine mortality and morbidity associated with diseases and injuries into one value. The DALY is calculated using disability weights estimated by the WHO, and for this study the weights used were 1 for cured TB (assumed perfect health), 0.729 for active TB (treated or untreated), 0.4 for blind and 0 for dead [36]. For example, if during the 10 years in the model, a patient lived with active TB for 2 years and then was cured and lived for another 8 years, then adjusting for disability, they would have lived for $2 \times 0.729$ (for active TB) $+8 \times 1$ (for cured state) $=9.46$ DALYs. During each 1-year cycle, if a patient died, defaulted, relapsed or became blind, we assumed the transition between the two corresponding Markov states (e.g. from active TB undergoing treatment to blind) occurred halfway during the year.

\section{TB treatment strategies}

The four unique treatment strategies and different treatment regimens examined in the model are summarised as follows.

\section{Reference treatment strategy (status quo): standard}

This consisted of initial treatment of new cases with the WHO-recommended 6-months standardised initial regimen (known as $2 \mathrm{HRZE} / 4 \mathrm{H} 3 \mathrm{R} 3$ ) consisting of 2 months of INH, RMP, pyrazinamide (PZA) and EMB taken daily (initial phase), followed by 4 months of INH and RMP taken three times per week (continuation phase). Patients who failed, relapsed after cure or defaulted received the WHO-recommended 
TABLE 1 Model parameters: values and sources\#

\section{Parameter}

Base case value

\section{Range used in \\ sensitivity \\ analysis}

Source

\section{Starting age years \\ TB-related mortality rates $\%$}

Drug-susceptible or mono/polyresistant TB Initial Std/EMB initial

Retreat Std/Str retreat/MDR Std

RMP-resistant/MDR-TB

Non-MDR-regimen

MDR Std

Other mortality rates

Untreated TB \%

Non-TB-related mortality \%

Mortality rate if blind

\section{Probability of EMB-induced blindness \%}

Initial Std

EMB initial/retreat Std/Str retreat

(EMB for 6-8 months)

MDR Std (EMB for 24 months)

\section{Treatment default rate ${ }^{+} \%$}

Drug-susceptible or mono/polyresistant TB

All initial regimens (6 months)

All 8-month retreatment regimens

MDR treatment

RMP-resistant/MDR-TB

Non-MDR-regimen

\section{MDR Std}

Treatment failure rate \%

Drug-susceptible TB

$$
\text { Any regimen }
$$

Mono/polyresistant TB

Initial Std/EMB initial

Retreat Std

Str retreat

MDR Std

RMP-resistant/MDR-TB

Non-MDR-regimen

\section{MDR Std}

\section{Probability of relapse \%}

Drug-susceptible TB

Any regimen

Mono/polyresistant TB

Initial Std/EMB initial

Retreat Std

Str retreat

MDR Std

RMP-resistant/MDR-TB

Non-MDR-regimen

MDR Std
Estimated based on cost surveys

\begin{tabular}{|c|c|c|}
\hline 36 & $18-60$ & Estimated based on cost surveys \\
\hline $\begin{array}{l}4 \\
7\end{array}$ & $\begin{array}{l}2-8 \\
4-11\end{array}$ & $\begin{array}{l}\text { WHO [27] } \\
\text { WHO [27] }\end{array}$ \\
\hline 33 & $30-40$ & Assumed same as untreated TB [28] \\
\hline 11 & $9-13$ & JoHNSTON et al. [29] \\
\hline 33 & $30-40$ & RIEDER [28] \\
\hline Age-specific & Age-specific & Used WHO life table for Ecuador [30] \\
\hline $\begin{array}{l}3.5 \times \text { age-specific } \\
\text { mortality rate }\end{array}$ & $\begin{array}{l}3.5 \times \text { age-specific } \\
\text { mortality rate }\end{array}$ & REMME [31] \\
\hline 0.0 & 0.0 & EMB in first 2 months assumed no blindness \\
\hline 0.23 & $0-0.61$ & EzER et al. [14] \\
\hline 0.46 & $0-0.99$ & $\begin{array}{c}\text { Assumed double the risk from retreatment due } \\
\text { to longer treatment duration }\end{array}$ \\
\hline 8 & $4-15$ & WHO [27] \\
\hline 15 & 4-30 & WHO [27] \\
\hline 14.7 & $11.2-19.0$ & Assumed same as MDR-TB [29] \\
\hline 0.0 & 0.0 & $\begin{array}{l}\text { Assumed that patients would only die, fail or be } \\
\text { spontaneously cured }\end{array}$ \\
\hline 14.7 & $11.2-19.0$ & JoHNSTON et al. [29] \\
\hline 0.3 & $0.1-0.4$ & MenZIES et al. [32] \\
\hline 2.8 & $0.7-14.7$ & MENZIES et al. [32] \\
\hline 7.4 & $0-15.1$ & MenZIES et al. [3] \\
\hline 3.7 & $0-15.1$ & $\begin{array}{l}\text { Assumed half the rate of first-line } \\
\text { retreatment [3] }\end{array}$ \\
\hline 0.4 & $0-1.5$ & Estimated from MeNZIES et al. [3] \\
\hline 42 & $35-45$ & $\begin{array}{l}\text { Assumed that patients could only die or be } \\
\text { spontaneously cured, and the remainder failed }[28,33]\end{array}$ \\
\hline 8 & $5-11$ & JoHNSTON et al. [29] \\
\hline 3.7 & $2.8-4.7$ & MenZIES et al. [32] \\
\hline 11.4 & $6.5-16.2$ & MeNZIES et al. [32] \\
\hline 4.6 & $4.0-14.5$ & Menzies et al. [3] \\
\hline 2.3 & $2.0-14.5$ & $\begin{array}{c}\text { Assumed half the rate of first-line } \\
\text { retreatment [3] }\end{array}$ \\
\hline 2.6 & $0-7.9$ & Estimated from MenzIES et al. [3] \\
\hline 2.5 & $1.3-2.5$ & $\begin{array}{l}\text { Reported relapse rate among untreated TB } \\
\text { cases who had spontaneous cures [33] }\end{array}$ \\
\hline 2.0 & $4.0-14.5$ & JoHNSTON et al. [29] \\
\hline
\end{tabular}




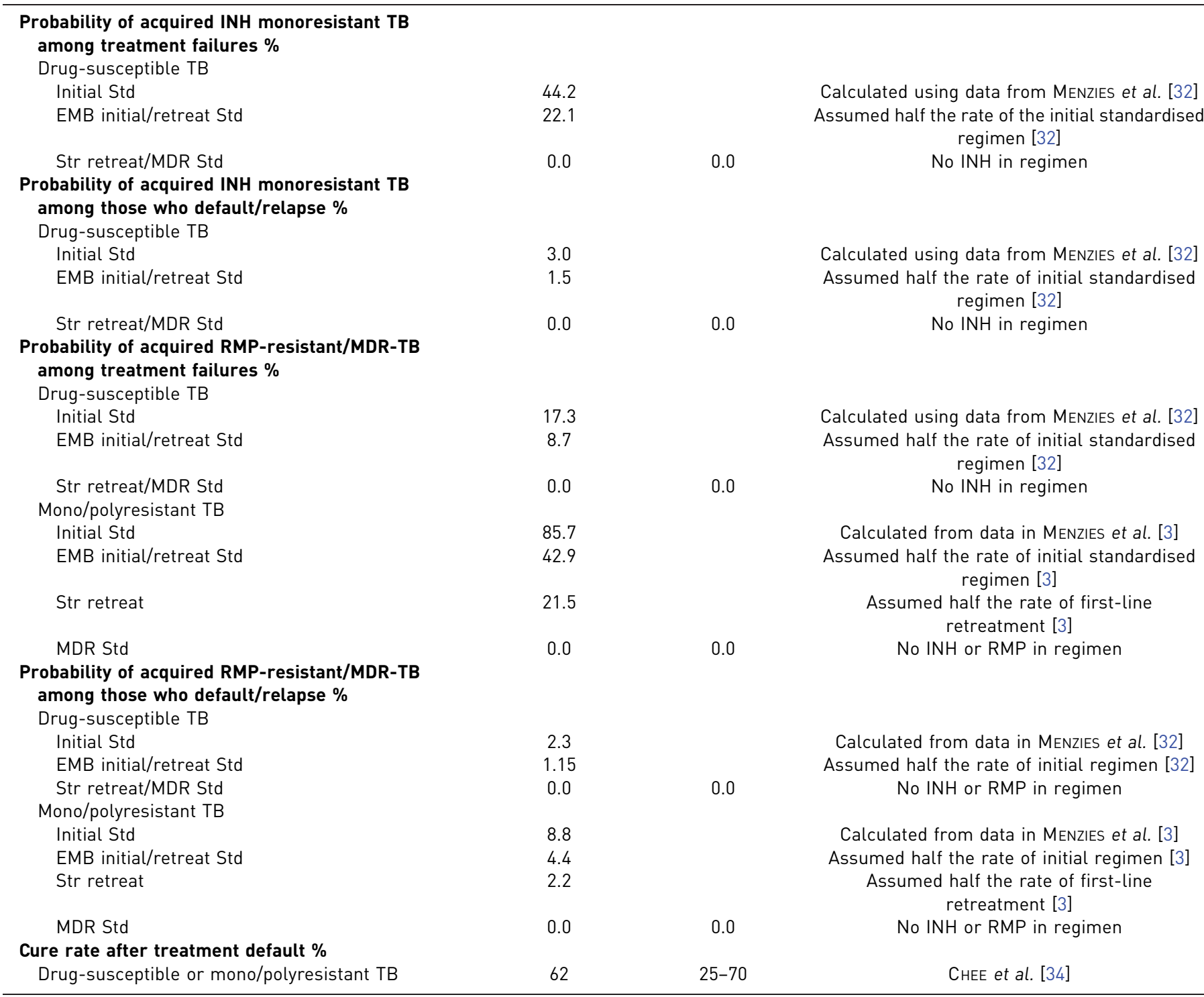

TB: tuberculosis; Std: standardised; EMB: ethambutol; Str retreat: strengthened retreatment; MDR: multidrug-resistant; RMP: rifampicin; WHO: World Health Organization; INH: isoniazid. " : all probabilities are based on end-of-treatment outcomes unless otherwise noted; ": we assumed that patients with MDR-TB not treated with the standardised MDR regimen experience only three treatment outcomes, fail, die or spontaneously cured; $0 \%$ of MDR-TB patients default; ${ }^{+}$: treatment default rates include patients who default treatment and those who transfer out. We assumed that treatment outcomes of transfers-out were equivalent to those of default [35].

standardised retreatment regimen with first-line drugs only (known as 2HRZES/1HRZE/5H3R3E3): streptomycin is given daily in the first 2 months; INH, RMP, PZA and EMB are taken daily for the first 3 months; and then INH, RMP and EMB are taken three times per week in the last 5 months.

\section{Ethambutol added to initial treatment: EMB initial}

All new patients received the WHO-recommended 6-month standardised initial regimen in settings with a high prevalence of INH resistance (2HRZE/4H3R3E3), consisting of 2 months of INH, RMP, PZA and EMB taken daily (initial phase), followed by 4 months of INH, RMP and EMB taken three times per week (continuation phase). Patients who failed, relapsed after cure or defaulted received the standardised 
retreatment regimen as in the standard strategy. Compared with the standard strategy, the addition of EMB throughout the initial regimen was assumed to reduce by $50 \%$ the probability of acquired INH monoresistance and acquired MDR-TB after initial treatment, and to increase the probability of EMBinduced blindness from $0 \%$ to $0.23 \%$, but did not change any other probabilities.

\section{Strengthened retreatment: Str retreat}

New patients received the same initial treatment as in the standard strategy, but those who failed or relapsed after cure or default received a retreatment regimen that included second-line drugs ( 2 months of daily levofloxacin, rifampicin, pyrazinamide, ethambutol and streptomycin, followed by 1 month of daily levofloxacin, rifampicin, pyrazinamide and ethambutol, then by 6 months of thrice-weekly levofloxacin, rifampicin, and ethambutol; 2LfxRZES/1LfxRZE/5Lfx3R3E3): for costing purposes (but without data confirming the efficacy of this regimen), we assumed the regimen would be an 8-month standardised regimen in which INH was replaced with levofloxacin.

Compared with the standard strategy the strengthened retreatment strategy was assumed to reduce by $50 \%$ the probability of failure, or relapse and associated acquired MDR-TB in retreated patients with initial INH resistance, but did not change any other probabilities.

\section{Standardised MDR treatment for failures of initial treatment: MDR failures}

New patients received the same initial treatment as in the standard strategy. Patients who defaulted or relapsed after cure from initial treatment received the same retreatment regimen as described for the standard strategy. But those who failed initial treatment received a 24-month standardised MDR regimen. All patients were hospitalised for only 1 month at the start of MDR treatment, given published studies of the effectiveness of community-based treatment [37-39]. Amikacin (Am) is injected daily for the first 6 months. PZA, EMB, levofloxacin and ethionamide are taken daily for 24 months (6AmZELfxEth/ 18ZELfxEth) [5, 27, 29].

Compared with the standard strategy, the MDR failures strategy (MDR failures) was assumed to improve treatment outcomes in failure cases who had MDR in order to achieve cure rates equivalent to published estimates using this regimen [3]. All other probabilities remained unchanged.

For the first three strategies, patients who failed, relapsed or defaulted after retreatment received the WHO recommended standardised MDR regimen (6AmZELfxEth/18ZELfxEth) [5]. For all four strategies, patients who failed, relapsed or defaulted after receiving one course of the MDR regimen were assumed to receive no further treatment, and to enter a state of untreated TB from which they could continue to live, die or be spontaneously cured. The annual spontaneous cure rate was assumed at $25 \%[28,33]$.

\section{Treatment outcomes and probabilities}

Estimates of transition probabilities and treatment outcomes, and published sources for these estimates are summarised in table 1.

There is no published information regarding the impact of adding EMB throughout initial treatment (EMB initial) on outcomes, and limited evidence on the treatment outcomes of a strengthened retreatment approach (Str retreat) where INH is replaced with a later generation fluoroquinolone regimens [40]. Hence, assumptions were made regarding their efficacy relative to the WHO standardised initial and retreatment regimens.

\section{Costs}

Costs were estimated from the societal perspective, and included health systems costs, patients' and families' out-of-pocket expenditure, and lost wages (online supplementary table S1). All costs were expressed in 2010 international US dollars, which were adjusted using 2007 power purchasing parity estimates for Ecuador [41], and inflated to 2010 currency using the average consumer price index in the US for 2007 and 2010 [42]. We used cost estimates from Ecuador because direct and indirect patient costs had been estimated there recently [43]. Indirect patient costs were based on patient and family time lost during $\mathrm{TB}$ diagnosis and treatment, which was converted into monetary costs using the hourly wage based on the power purchasing parity-adjusted average per capita gross national income [44].

Ecuador has one of the highest levels of MDR-TB in Latin America [2], which makes it an appropriate setting for estimating MDR-TB treatment costs. The country achieved national implementation of directly observed therapy, short course (DOTS) in 2006, and provides TB treatment and diagnosis free of charge for patients $[45,46]$. Thus, estimated treatment costs should be comparable with other countries that have national coverage for DOTS. 
Estimating health system costs

An interviewer-administered health facility cost survey was used to collect health system data in Ecuador. The interviews were conducted in 19 urban health facilities that treated TB patients in two provinces (Guayas and Tungurahua) between September and October 2009: 16 Ministry of Health clinics, two Ministry of Health hospitals and one nongovernment hospital (Erika Leung, McGill University, Montreal, Canada; personal communication). Salary levels of Ministry of Health employees were retrieved from the Ministerio de Inclusión Económica y Social [47]. We assumed that the personnel costs made up $80 \%$ of the total facility budget $[48,49]$. The average health system cost per outpatient visit was the quotient of dividing the estimated total facility budget of each Ministry of Health clinic by the reported number of annual outpatient visits.

We assumed that the total inpatient budget of a hospital was the difference between the total health facility budget and the total outpatient budget. The per diem cost was the total inpatient budget divided by annual number of bed-days. Only data from one Ministry of Health hospital, Hospital Alfredo J. Valenzuela, was included because it was a respiratory/pulmonary-specific hospital and would be more applicable for estimating TB-related per diem costs. The cost of each directly observed therapy visit was estimated to be a third of the cost of an outpatient visit. This assumption was based on previously published surveys of health system costs $[50,51]$.

\section{Cost-effectiveness analyses}

We calculated three incremental cost-effectiveness ratios (ICERs): cost per MDR case averted, cost per death averted and cost per DALY gained.

\section{Sensitivity analyses}

We performed one-way sensitivity analyses to account for uncertainties in key model assumptions: prevalence of INH and MDR monoresistance; the probability of EMB-induced blindness; the efficacy of adding EMB throughout initial treatment and of strengthening the retreatment regimen with a second-line drug; the duration of hospitalisation during MDR treatment; and varying the discount rates used. The ranges chosen for each analysis were based on the published literature, WHO reports or expert opinion. We also performed probabilistic sensitivity analysis to incorporate statistical or sampling variability in input parameter estimates by performing 10000 Monte Carlo simulation trials per analysis. We used 95\% confidence intervals or ranges to estimate corresponding probability distributions with the method of moments approach [52]. $\gamma$ distributions were assigned to cost parameters and $\beta$ distributions to probability estimates [52]. Using the results from the probabilistic sensitivity analyses, cost-effectiveness acceptability curves were constructed to compare the different treatment strategies in the four hypothetical settings. These curves plot the probability that a given treatment strategy is cost-effective compared to the standard WHO strategy given a maximum acceptable ICER threshold. This threshold is also known as the willingness-to-pay threshold, indicating the maximum amount the group of interest (patient, government or society) is willing to pay per unit of benefit gained.

\section{Results}

\section{Treatment-related outcomes}

With the standard strategy, we predicted between 407 and 781 new MDR cases from acquired drug resistance, between $1.3 \%$ and $7.3 \%$ failures, and between $5.2 \%$ and $9.8 \%$ deaths, depending upon whether the prevalence of drug resistance was low or high (table 2). All three alternative treatment strategies resulted in fewer cases of MDR-TB and fewer TB deaths than standard (tables 2 and 3).

Overall, EMB initial was predicted to avert the most cases of MDR-TB compared with standard. This strategy was predicted to prevent between 337 and 347 MDR cases per 100000 patients when the prevalence of INH monoresistant TB was 15\%, depending on whether the prevalence of MDR-TB was high (10\%) or low (1\%), respectively. When the prevalence of INH-resistant TB was $5 \%$, this strategy was predicted to avert 177 and 186 MDR cases when the prevalence of MDR-TB was $10 \%$ and $1 \%$, respectively. The other strategies were predicted to avert fewer MDR cases: between 30 and $61 \mathrm{MDR}-\mathrm{TB}$ cases for the strengthened retreatment strategy, and only five or six cases per 100000 patients for the MDR failure strategy.

MDR failure strategy averted the most TB-related deaths compared with the standard strategy in all settings (between 75 to 664 deaths averted per 100000 patients) except when the prevalence of INH monoresistant was $15 \%$ and the prevalence of MDR-TB was $1 \%$, in which case the EMB initial strategy averted the most deaths at 128 per 100000 patients.

The EMB initial strategy consistently resulted in a loss of DALYs compared with the standard strategy. Conversely, strengthened retreatment and MDR failure resulted in gains in DALYs compared with standard 


\begin{tabular}{|c|c|c|c|c|}
\hline 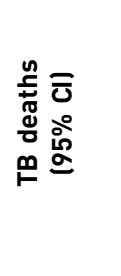 & 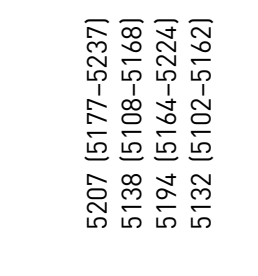 & 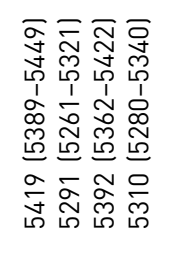 & 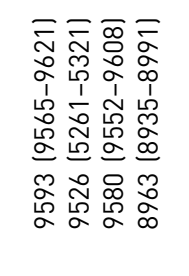 & 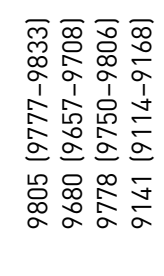 \\
\hline 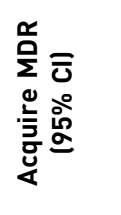 & 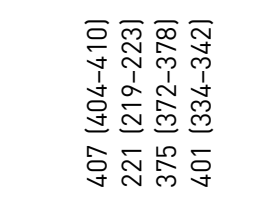 & 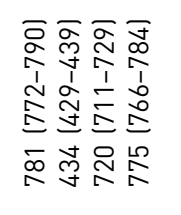 & 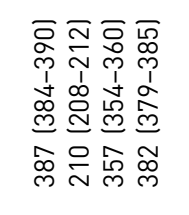 & 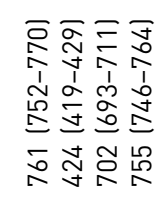 \\
\hline 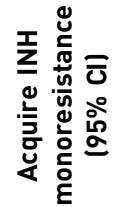 & 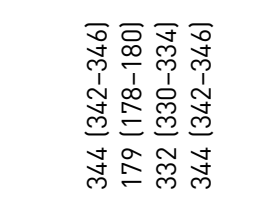 & 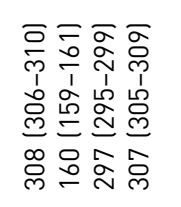 & 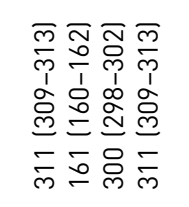 & 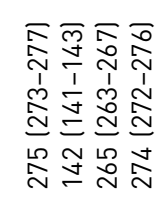 \\
\hline 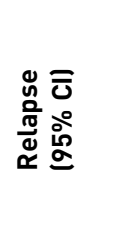 & 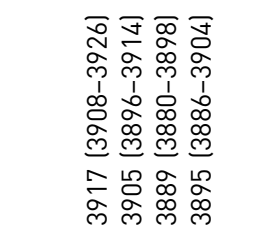 & 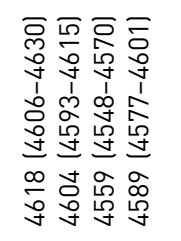 & 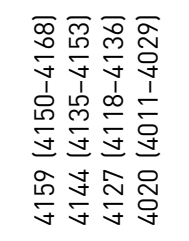 & 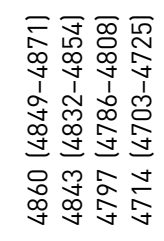 \\
\hline 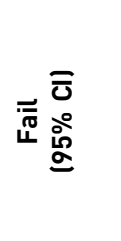 & 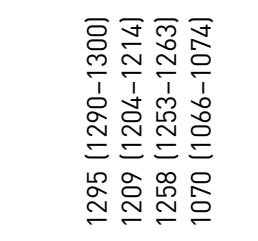 & 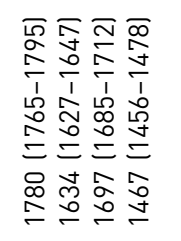 & 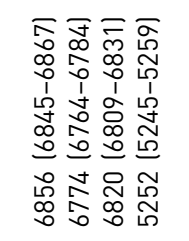 & 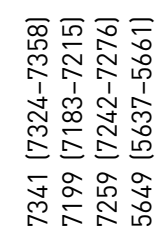 \\
\hline 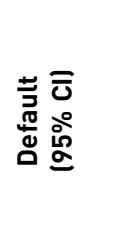 & 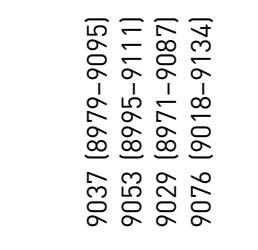 & 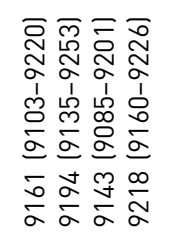 & 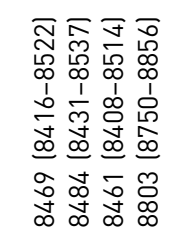 & 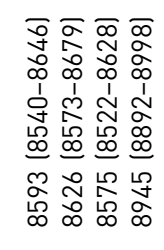 \\
\hline 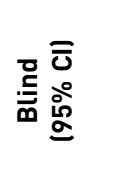 & 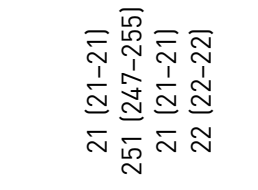 & 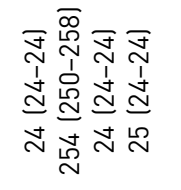 & 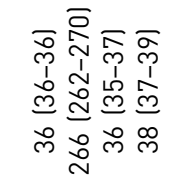 & 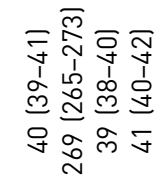 \\
\hline 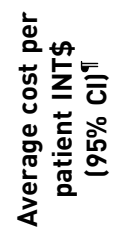 & 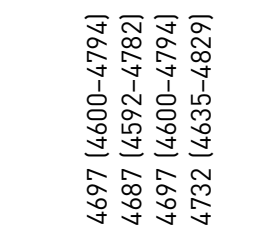 & 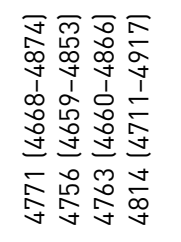 & 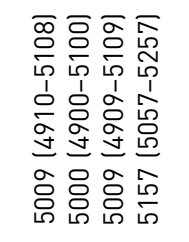 & 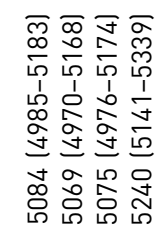 \\
\hline 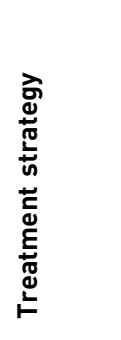 & 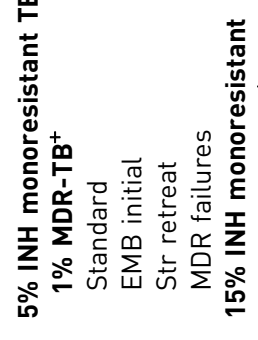 & . & 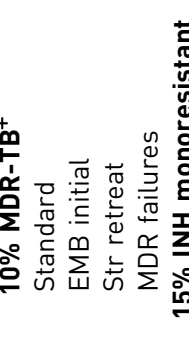 & \\
\hline
\end{tabular}




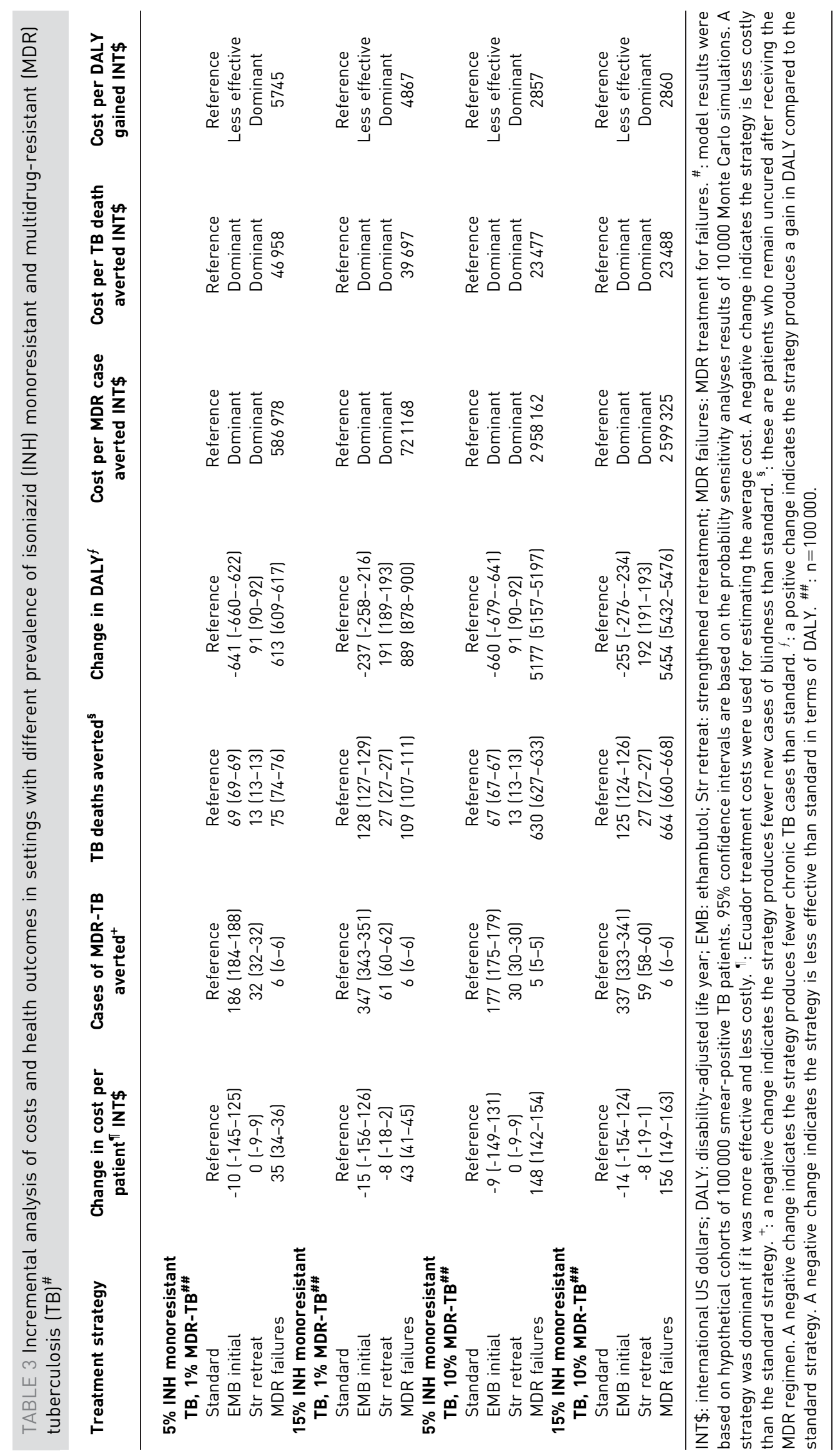


in all four settings. MDR failures resulted in the largest gains in DALYs in all settings, from 613 to 5454 total DALYs gained per 100000 patients compared with standard regimen, and the gains were larger when the prevalence of MDR-TB was high at $10 \%$ and largest when both prevalence of MDR-TB and mono-INH resistant TB were high.

\section{Treatment-related costs}

Standardised MDR treatment of all failures of initial treatment (MDR failures) was the most costly treatment strategy in all four settings. The EMB initial and strengthened retreatment strategies were equal in cost or less costly than standard in all four settings (tables 2 and 3). EMB initial was the least costly of all four strategies in all settings.

\section{Incremental cost-effectiveness ratios}

The strengthened retreatment strategy dominated the standard strategy, the more effective and less costly strategy, in terms of cost per MDR case averted, cost per TB death averted and cost per DALY gained compared with the standard treatment strategy in all four settings (table 2 and 3). EMB initial dominated standard as well as strengthened retreatment strategies, resulting in more cost savings and fewer MDR cases and TB deaths than both strategies. However, these advantages were offset by the predicted development of blindness in some patients, so that this strategy resulted in fewer total DALYs than the standard strategy (i.e. was less effective) in all four settings.

The MDR failures strategy cost between $\$ 2857$ and $\$ 2860$ per DALY gained compared with standard when the prevalence of MDR-TB was high at $10 \%$. The cost per DALY gained increased when the prevalence of MDR-TB was low at $1 \%$, between $\$ 4867$ and $\$ 5745$ per DALY gained compared with standard. A similar trend was observed for cost per TB death averted, and the opposite for cost per MDR case averted.

TABLE 4 One-way sensitivity analysis of individual parameters in the model ${ }^{\#}$

\begin{tabular}{|c|c|c|c|}
\hline Treatment strategy & $\begin{array}{l}\text { Cost per MDR case averted } \\
\text { INT\$ }\end{array}$ & $\begin{array}{c}\text { Cost per TB death averted } \\
\text { INT\$ }\end{array}$ & $\begin{array}{l}\text { Cost per DALY gained } \\
\text { INT\$ }\end{array}$ \\
\hline
\end{tabular}

\begin{tabular}{|c|c|c|c|}
\hline \multicolumn{4}{|c|}{$\begin{array}{l}\text { Prevalence of INH monoresistant TB among } \\
\text { new TB patients }(5-60 \%)\end{array}$} \\
\hline \multicolumn{4}{|c|}{$1 \%$ MDR-TB } \\
\hline Standard & Reference & Reference & Reference \\
\hline EMB initial & Always dominant & Always dominant & $\begin{array}{l}\text { Less effective to dominant } \\
\qquad(>21 \%)\end{array}$ \\
\hline Str retreat & Always dominant & Always dominant & Always dominant \\
\hline MDR failures & $639962-795014$ & $52456-30460$ & 6387-3717 \\
\hline \multicolumn{4}{|l|}{$10 \%$ MDR-TB } \\
\hline Standard & Reference & Reference & Reference \\
\hline EMB initial & Always dominant & Always dominant & $\begin{array}{l}\text { Less effective to dominant } \\
\qquad(>21 \%)\end{array}$ \\
\hline Str retreat & Always dominant & Always dominant & Always dominant \\
\hline MDR failures & $3617188-1921908$ & $23450-23553$ & $2856-2867$ \\
\hline \multicolumn{4}{|c|}{$\begin{array}{l}\text { Prevalence of MDR-TB among new TB patients } \\
(1-20 \%)\end{array}$} \\
\hline \multicolumn{4}{|c|}{ 5\% Mono-INH resistant TB } \\
\hline Standard & Reference & Reference & Reference \\
\hline EMB initial & Always dominant & Always dominant & Always less effective \\
\hline Str retreat & Always dominant & Always dominant & Always dominant \\
\hline MDR failures & $586978-6827963$ & $46958-21885$ & 5745-2665 \\
\hline \multicolumn{4}{|c|}{$15 \%$ Mono-INH resistant TB } \\
\hline Standard & Reference & Reference & Reference \\
\hline EMB initial & Always dominant & Always dominant & Always less effective \\
\hline Str retreat & Always dominant & Always dominant & Always dominant \\
\hline MDR failures & $721168-4686166$ & $39697-21932$ & 4867-2671 \\
\hline
\end{tabular}

Data are presented as incremental cost-effectiveness ratio (low-high). MDR: multidrug-resistant; INT\$: international US dollars; TB: tuberculosis; DALY: disability-adjusted life year; INH: isoniazid; EMB: ethambutol; Str retreat: strengthened retreatment; MDR failures: MDR treatment for failures. ": a strategy was dominant if it was more effective and less costly. The incremental cost-effectiveness ratio ranges correspond to results when the input parameter is varied from low to high. If the incremental cost-effectiveness changes from a number to either "dominant" or "less effective", or vice versa, the threshold values of the corresponding input parameter are indicated in parentheses. ": Ecuador treatment costs were used for estimating the average cost. 


\section{Sensitivity analyses}

Prevalence of drug resistance was an important determinant of cost-effectiveness as shown in table 4. EMB initial was always dominant (less costly and more effective) compared with the standard strategy in terms of cost per MDR cases averted and cost per death averted regardless of the drug resistance prevalence. However, it was less effective in terms of cost per DALY gained compared to standard under most conditions, but dominated standard when the prevalence of INH monoresistant TB was $>21 \%$. Conversely, strengthened retreatment was always dominant over standard. The MDR failure strategy never dominated standard; however, it was always more effective. It became more cost-effective as the prevalence of MDR-TB increased, and bacame as low as $\$ 2665$ per DALY gained when the prevalence of INH monoresistant TB was $5 \%$ and that of MDR-TB was $20 \%$.

Strengthened retreatment was consistently dominant over standard treatment when other key assumptions were changed, except that it became more costly than standard when the efficacy of the strengthened retreatment regimen was less than approximately two times that of the standard retreatment, and when the hospitalisation period for MDR treatment was $<1$ month long, in settings with $5 \%$ INH monoresistant TB (online supplementary table S2). MDR failure was also more cost-effective when the duration of hospitalisation during MDR treatment was shorter, being as low as \$664 per DALY gained when there was no hospitalisation, and was less cost-effective when it was long, being as high as \$25044 per DALY gained when there were 6 months of hospitalisation. Findings remained similar when we used the WHOrecommended discount rates of $6 \%$ for costs and $0 \%$ for health outcomes.

Results of probabilistic sensitivity analyses are shown in figure 2. The incremental costs of the EMB initial strategy varied widely, as did DALYs gained or lost, although in the majority of the simulations this strategy led to fewer DALYs than the standard strategy. Conversely, the strengthen retreatment and MDR failure strategies consistently led to more DALYs, with a much narrower range of incremental costs, centred around no difference, compared with the standard strategy.

Based on these analyses, cost-effectiveness acceptability curves comparing the three individual strategies to the baseline strategy, standard, were created (online supplementary fig. S1). EMB initial was least likely to be cost-effective in terms of cost per DALY gained compared with standard among the three alternative strategies in all settings; its probability of cost-effectiveness ranged from $11.7 \%$ to $23.7 \%$. After accounting for statistical uncertainty, MDR failures becomes more likely to be cost-effective compared with standard than both strengthened retreatment and EMB initial as the willingness-to-pay amount per DALY gained increases. However, strengthened retreatment is more likely to be cost-effective when the willingness-to-pay amount is as follows: below $\$ 4848$ per DALY gained for low prevalence of INH monoresistance (5\%) and MDR-TB (1\%); below $\$ 4560$ per DALY gained for high INH monoresistance (15\%) and low MDR-TB prevalence; below $\$ 2448$ per DALY gained for low INH monoresistance and high MDR-TB (10\%) prevalence; and below \$2648 per DALY gained for high INH monoresistance and high MDR-TB prevalence.

\section{Discussion}

This study provides evidence that a treatment strategy incorporating a strengthened retreatment regimen containing at least one second-line drug could lead to both cost savings and increased treatment effectiveness in terms of DALYs gained, MDR-TB cases averted and TB-related deaths averted compared with one using the standard WHO retreatment regimen. This finding was true in settings with varying prevalence of drug resistance, and in several one-way sensitivity analyses. However, despite the robustness of the finding, this treatment strategy would avert fewer MDR cases and lead to less cost saving than adding EMB to the initial treatment, and would avert fewer TB deaths than either adding EMB to initial treatment or giving a standardised MDR regimen to failures of initial treatment.

We estimated that adding EMB throughout the initial standardised treatment would lead to a loss of 237660 DALYs per 100000 patients compared with the standardised WHO initial treatment in four settings with widely different prevalence of drug resistance. This suggests that the benefits of averting MDR-TB cases and related deaths may be outweighed by the disability endured by a larger number of patients who develop blindness. However, we found that if the assumed risk of developing blindness from 6-8 months of EMB treatment was lower than $\sim 0.1 \%$, then adding EMB to the initial standardised regimen would lead to gains in DALYs, with fewer MDR-TB and deaths, and net cost savings compared with the standard initial regimen. A recently published systematic review and meta-analysis estimated that the risk of EMB-induced blindness was $0.23 \%$ (95\% CI $0.0-0.61 \%$ ) [14]. Given these confidence intervals, it is possible that the health gains of adding EMB could outweigh the risk of blindness in settings with a higher prevalence of INH resistance.

To assess one of the new treatment recommendations of the WHO [15], we examined the costs and treatment outcomes of empirically treating all patients who failed initial treatment with an MDR regimen. 
a)
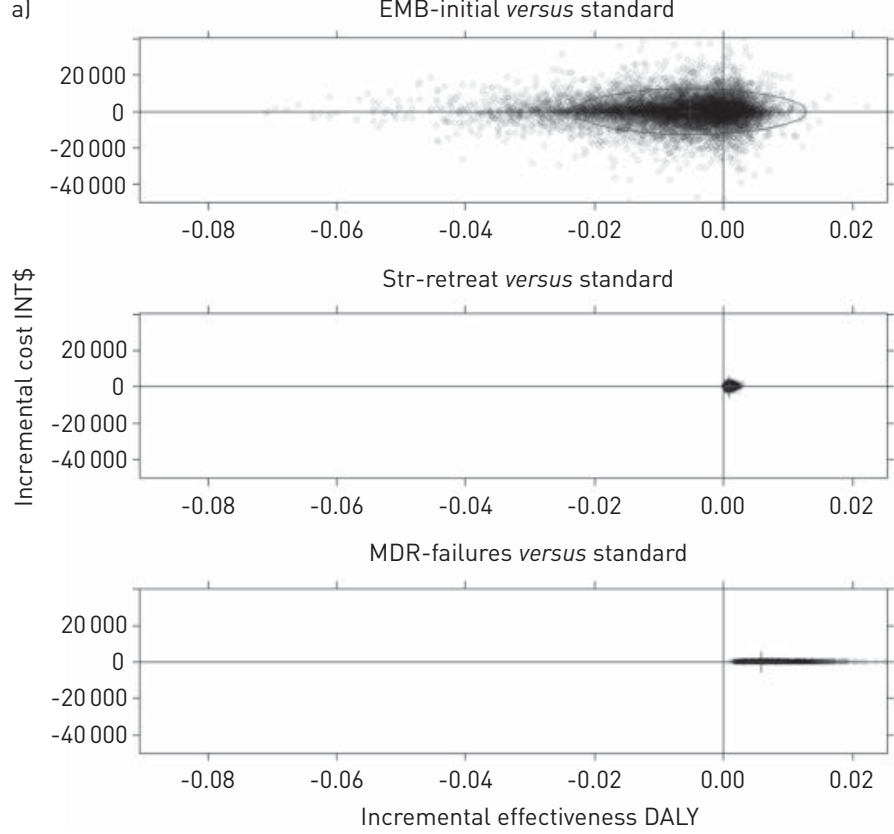

c)
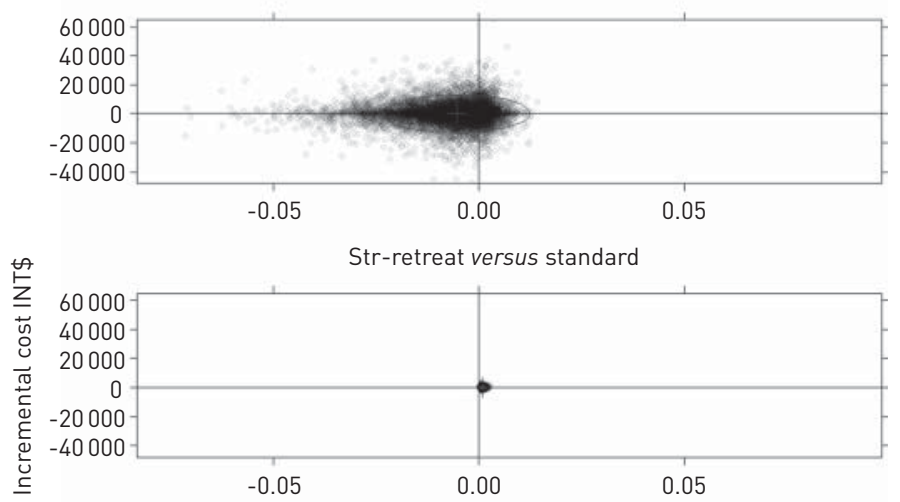

MDR-failures versus standard

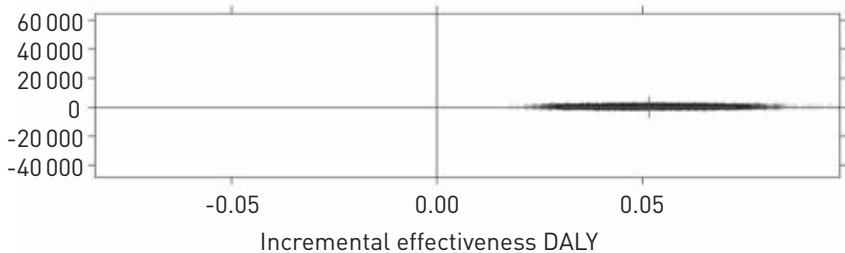

b)
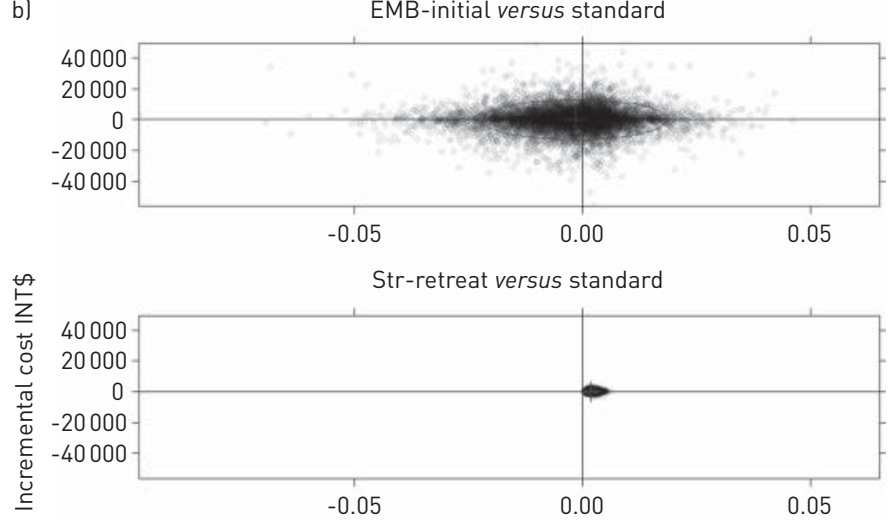

MDR-failures versus standard

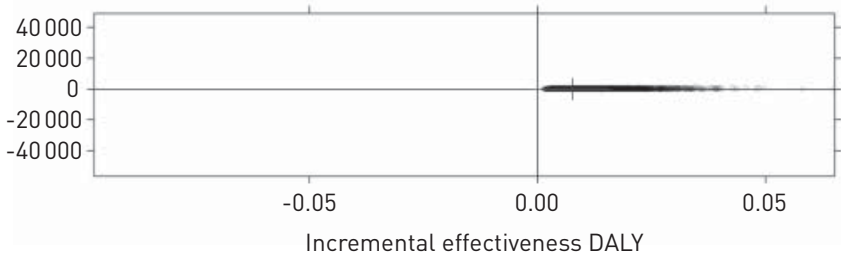

d)
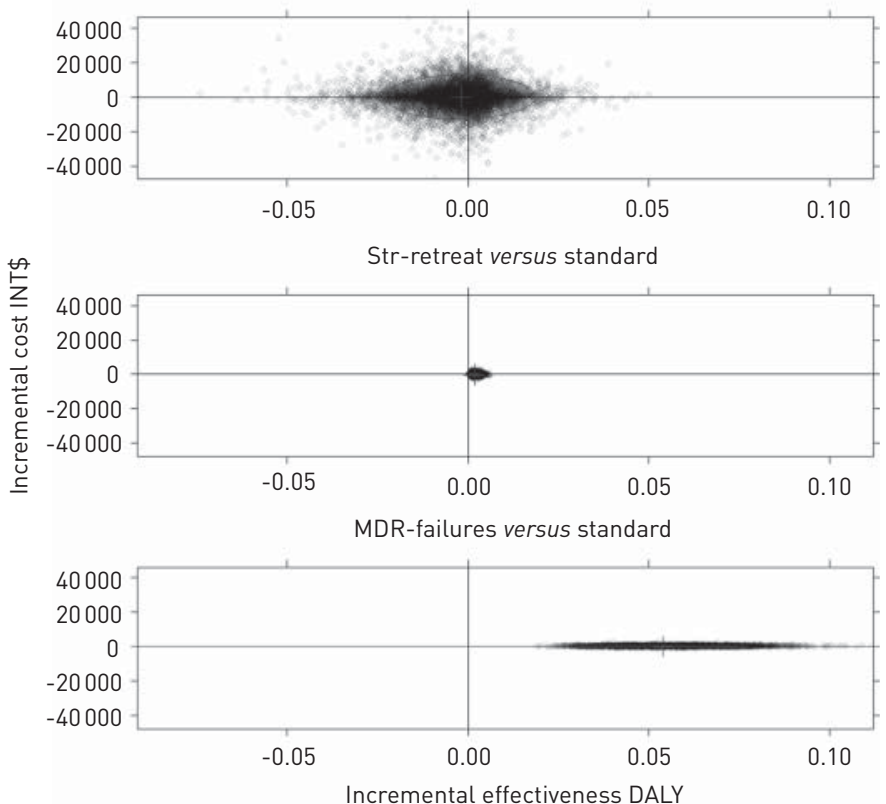

FIGURE 2 Probability sensitivity analyses of incremental cost versus incremental change in average disability-adjusted life years (DALYs) per patient, comparing alternative treatment strategies to standard. The analyses were performed using 10000 second-order Monte Carlo simulation trials in the four settings. a) 5\% isoniazid (INH) monoresistant tuberculosis (TB) and 1\% multidrug-resistant (MDR)-TB; b) $15 \%$ INH monoresistant TB and $1 \%$ MDR-TB; c) 5\% INH monoresistant TB and 10\% MDR-TB; and d) 15\% INH monoresistant TB and 10\% MDR-TB. Average DALYs gained or lost per patient comparing each strategy (ethambutol (EMB) initial, strengthened retreatment (Str retreat) and MDR failures) to standard is shown on the $x$-axis, and the change in average cost per patient is shown on the $y$-axis. Each black circle represents the result of one simulation trial. The ellipse depicts the $95 \%$ confidence interval of the incremental cost and incremental effectiveness estimates. INT\$: international US dollars.

Comparing this MDR failure strategy with the standard treatment strategy, the cost per DALY gained ranged from $\$ 2857$ to $\$ 5745$. This would be considered cost-effective in most middle-income countries that have a per capita gross national income above that range, a commonly used ICER threshold [53-56]. This strategy was also the most likely to be cost-effective compared with the other alternative strategies if the willingness to pay ICER threshold exceeded $\$ 4560$ per DALY gained. 


\section{Limitations of the study}

There are several limitations of this study. In our model, we analysed closed cohorts of TB patients and did not model disease transmission. However, this would probably result in an underestimate of the benefits of treatment strategies that averted MDR-TB cases, as this would also prevent transmission of drug-resistant strains of TB. A recently published systematic review of the impact of TB on health-related quality of life reported that longer duration of TB disease, or having MDR-TB, were associated with poorer health-related quality of life [57]. As we did not account for these differences in our model, it is possible that the average DALYs per patient were overestimated, particularly for patients who received more than one treatment, or had MDR-TB. Furthermore, there is likely to be more morbidity associated with the MDR regimen compared with other treatment regimens than accounted for by a uniform disability weight applied to all patients with active TB, regardless of treatment. Thus, the effectiveness of treating failures from initial treatment with an MDR regimen could have been overestimated.

Estimates of treatment outcomes were taken from systematic reviews and meta-analyses whenever possible in order to improve precision, and reduce potential biases from selecting a small sample of individual studies known to the investigators. However, there was little published evidence for the treatment outcomes of the alternative regimens we considered. We conservatively assumed that a retreatment regimen strengthened with a second-line drug would not improve treatment outcomes for patients with MDR-TB compared with the standard WHO retreatment regimen; however, this could have underestimated the effectiveness of using the strengthened retreatment regimen. Furthermore, our estimated treatment-related costs were based on studies conducted in Ecuador, which may not be generalised to other settings, particularly those with different $\mathrm{TB}$ treatment practices and from different income settings. Hence, we examined these uncertainties by performing extensive sensitivity analyses. In addition, we were not able to explicitly consider HIV in the model due to the paucity of published estimates of treatment outcomes in HIV co-infected patients with drug-resistant TB treated with various regimens. Some studies have previously shown that co-infected patients may experience greater risks of relapse and mortality, while others have not found any significant differences in treatment outcome [58-61].

\section{Policy implications}

This study has shown that the prevention and treatment of drug-resistant TB is complex, and requires careful consideration of health benefits, costs and treatment-related morbidities, such as EMB-induced blindness. This study also found that empirical treatment of all patients who fail initial treatment with an MDR regimen could avert a large number of TB-related deaths. However, this would be at the cost of treating many patients with lengthy and toxic MDR treatment who would not need this. Furthermore, this strategy would not avert MDR-TB and would have little additional societal impact. The analyses presented in this study provide supportive evidence for strengthening the standard retreatment regimen. This strategy could potentially lead to cost savings, and avert MDR-TB cases and TB-related deaths, even in settings with relatively low prevalence of INH monoresistant TB.

\section{Acknowledgements}

We would like to thank R. Steffen (Federal University of Rio de Janeiro, Rio de Janiero, Brazil), and V. Rouzier and E. Leung (both McGill University, Montreal, Canada) for collecting and providing data used in this study.

\section{References}

1 World Health Organization. Global Tuberculosis Report 2012. www.who.int/tb/publications/global_report/en/ index.html Date last accessed: August 17, 2013.

2 World Health Organization. Anti-Tuberculosis Drug Resistance in the World: Fourth Global Report. Report number WHO/HTM/TB/2008.39 http://whqlibdoc.who.int/hq/2008/WHO_HTM_TB_2008.394_eng.pdf Date last accessed: August 17, 2013.

3 Menzies D, Benedetti A, Paydar A, et al. Standardized treatment of active tuberculosis in patients with previous treatment and/or with mono-resistance to isoniazid: a systematic review and meta-analysis. PLoS Med 2009; 6: $1-14$.

4 Lew W, Pai M, Oxlade O, et al. Initial drug resistance and tuberculosis treatment outcomes: systematic review and meta-analysis. Ann Intern Med 2008; 149: 123-134.

5 World Health Organization. Towards universal access to diagnosis and treatment of multidrug-resistant and extensively drug-resistant tuberculosis by 2015: WHO progress report 2011. www.who.int/tb/publications/2011/ mdr_report_2011/en/ Date last accessed: August 17, 2013.

6 Skrahina A, Hurevich H, Zalutskaya A, et al. Alarming levels of drug-resistant tuberculosis in Belarus: results of a survey in Minsk. Eur Respir J 2012; 39: 1425-1431.

7 World Health Organization. Treatment of tuberculosis: guidelines for national programmes. 4th Edn. Report number WHO/HTM/TB/2009.420. www.who.int/tb/publications/tb_treatmentguidelines/en/ Date last accessed: August 17, 2013.

8 Kim Y, Suh G, Chung M, et al. Treatment of isoniazid-resistant pulmonary tuberculosis. BMC Infect Dis 2008; 8: 6 .

9 Koh W, Kwon O, Park Y, et al. Development of multidrug resistance during treatment of isoniazid-resistant tuberculosis. Eur Respir J 2005; 26: 557. 

Chest 1986; 80: 288-291.

Harcombe A, Kinnear W, Britton J, et al. Ocular toxicity of ethambutol. Respir Med 1991; 85: 151-153.

Kahana L. Toxic ocular effects of ethambutol. CMAJ 1987; 137: 213-216.

Leibold J. The ocular toxicity of ethambutol and its relation to dose. Ann NY Acad Sci 1966; 135: 904-909.

Ezer N, Benedetti A, Darvish-Zargar M, et al. Incidence of ethambutol-related visual impairment during treatment of active tuberculosis. Int J Tuberc Lung Dis 2013; 17: 447-455.

15 Falzon D, Jaramillo E, Schunemann HJ, et al. WHO guidelines for the programmatic management of drug-resistant tuberculosis: 2011 update. Eur Respir J 2011; 38: 516-528.

16 Weinstein MC, Siegel JE, Gold MR, et al. Recommendations of the panel on cost-effectiveness in health and medicine. JAMA 1996; 276: 1253-1258.

17 Drummond MF, Sculpher MJ, Torrance GW, et al. Methods for the Economic Evaluation of Health Care Programmes, Oxford: Oxford University Press, 2005.

18 Khan FA, Minion J, Pai M, et al. Treatment of active tuberculosis in HIV-coinfected patients: a systematic review and meta-analysis. Clin Infect Dis 2010; 50: 1288-1299.

19 Baltussen R, Floyd K, Dye C. Cost effectiveness analysis of strategies for tuberculosis control in developing countries. BMJ 2005; 331: 1364.

20 Sterling TR, Lehmann HP, Frieden TR. Impact of DOTS compared with DOTS-plus on multidrug resistant tuberculosis and tuberculosis deaths: decision analysis. BMJ 2003; 326: 574

21 Wells CD, Cegielski JP, Nelson LJ, et al. HIV infection and multidrug-resistant tuberculosis: the perfect storm. J Infect Dis 2007; 196: Suppl. 1, S86-S107.

22 Telenti A, Imboden P, Marchesi F, et al. Detection of rifampicin-resistance mutations in Mycobacterium tuberculosis. Lancet 1993; 341: 647-651.

23 Mokaddas E, Ahmad S, Samir I. Secular trends in susceptibility patterns of Mycobacterium tuberculosis isolates in Kuwait, 1996-2005. Int J Tuberc Lung Dis 2008; 12: 319-325.

24 Van Rie A, Warren R, Mshanga I, et al. Analysis for a limited number of gene codons can predict drug resistance of Mycobacterium tuberculosis in a high-incidence community. J Clin Microbiol 2001; 39: 636-641.

25 Siddiqi N, Shamim M, Hussain S, et al. Molecular characterization of multidrug-resistant isolates of Mycobacterium tuberculosis from patients in North India. Antimicrob Agents Chemother 2002; 46: 443-450.

26 Afanas'ev MV, Ikryannikova LN, Il'ina EN, et al. Molecular characteristics of rifampicin- and isoniazid-resistant Mycobacterium tuberculosis isolates from the Russian Federation. J Antimicrob Chemother 2007; 59: 1057-1064.

27 World Health Organization. Global tuberculosis control: epidemiology, strategy, financing: WHO report 2009. Report number WHO/HTM/TB/2009.411. www.who.int/tb/publications/global_report/2009/en/ Date last accessed: August 17, 2013.

28 Rieder HL. Epidemiologic Basis of Tuberculosis Control, Paris: International Union Against Tuberculosis and Lung Disease, 1999.

29 Johnston JC, Shahidi NC, Sadatsafavi M, et al. Treatment outcomes of multidrug-resistant tuberculosis: a systematic review and meta-analysis. PLoS One 2009; 4: e6914.

30 World Health Organization. Global Health Observatory Data Repository - Life expectancy: Life Tables Ecuador. Geneva, WHO, 2009. http://apps.who.int/gho/data/view.main.60490?lang=en. Date last accessed: August 17, 2013. Remme JHF. The Global Burden of Onchocerciasis in 1990, Geneva: World Health Organization, 2004.

32 Menzies D, Benedetti A, Paydar A, et al. Effect of duration and intermittency of rifampin on tuberculosis treatment outcomes: a systematic review and meta-analysis. PLoS Med 2009; 6: e1000146.

33 Grzybowski S, Enarson DA. The fate of cases of pulmonary tuberculosis under various treatment programmes. Bull Int Union Tuberc 1978; 53: 70-74.

34 Chee CBE, Boudville IC, Chan SP, et al. Patient and disease characteristics, and outcome of treatment defaulters from the Singapore TB control unit-a one-year retrospective survey. Int J Tuberc Lung Dis 2000; 4: 496-503.

35 Cummings KC, Mohle-Boetani J, Royce S, et al. Movement of tuberculosis patients and the failure to complete antituberculosis treatment. Am J Respir Crit Care Med 1998; 157: 1249-1252.

36 Murray CJL, Lopez AD. The Global Burden of Disease: A Comprehensive Assessment of Mortality and Disability from Diseases, Injuries, and Risk Factors in 1990 and Projected to 2020, Cambridge: Harvard University Press, 1996.

37 Van Deun A, Salim MAH, Das APK, et al. Results of a standardised regimen for multidrug-resistant tuberculosis in Bangladesh. Int J Tuberc Lung Dis 2004; 8: 560-567.

38 Nathanson E, Lambregts-van Weezenbeek C, Rich ML, et al. Multidrug-resistant tuberculosis management in resource-limited settings. Emerg Infect Dis 2006; 12: 1389-1397.

39 Tupasi TE, Gupta R, Quelapio MID, et al. Feasibility and cost-effectiveness of treating multidrug-resistant tuberculosis: a cohort study in the Philippines. PLoS Med 2006; 3: e352.

40 Dorman SE, Johnson JL, Goldberg S, et al. Substitution of moxifloxacin for isoniazid during intensive phase treatment of pulmonary tuberculosis. Am J Respir Crit Care Med 2009; 180: 273-280.

41 Heston A, Summers R, Aten B. Penn World Table Version 6.3, Center for International Comparisons of Production, Income and Prices at the University of Pennsylvania, August 2009. http://pwt.econ.upenn.edu/ php_site/pwt_index.php Date last accessed: August 17, 2013.

42 US Dept of Labor Bureau of Labor Statistics. Consumer Price Index - All Urban Consumers (CPI - U). ftp://ftp.bls. gov/pub/special.requests/cpi/cpiai.txt Date last accessed: August 17, 2013.

43 Rouzier VA, Oxlade O, Verduga R, et al. Patient and family costs associated with tuberculosis, including multidrug resistant tuberculosis in Ecuador. Int J Tuberc Lung Dis 2010; 14: 1316-1322.

44 World Bank. World Development Indicators database, Washington, DC: World Bank, 2010.

45 Vaca J, Peralta H, Gresely L, et al. DOTS implementation in a middle-income country: development and evaluation of a novel approach. Int J Tuberc Lung Dis 2005; 9: 521-527.

46 The Global Fund. Expansion of DOTS Strategy in Ecuador, Geneva, The Global Fund to FIght AIDS, Tuberculosis and Malaria, 2011. 

www.correosdelecuador.com.ec/_upload/TABLA\%20DE\%20REMUNERACI\%C3\%93N\%20SALARIAL\%202010.pdf Date last accessed: December 5, 2013.

48 Deficiencias en los hospitales son nacionales [National deficiencies in the hospitals]. Ecuadorinmediato.com. www.ecuadorinmediato.com/index.php? module $=$ Noticias\&func $=$ news_user_view\&id $=35229 \& u m t=$ expreso guayaquil_deficiencias_en_hospitales_son_nacionales Date last accessed: December 5, 2013.

49 Emergencia en el hospital delfina torres de conha. [Emergency in the Hospital Delfina Torres de Concha]. August 16, 1998. www.explored.com.ec/noticias-ecuador/emergencia-en-el-hospital-delfina-torres-de-conha-95071-95071. html Date last accessed: August 17, 2013.

50 Aspler A, Menzies D, Oxlade O, et al. Cost of tuberculosis diagnosis and treatment from the patient perspective in Lusaka, Zambia. Int J Tuberc Lung Dis 2008; 12: 928-935.

51 Jacquet V, Morose W, Schwartzman K, et al. Impact of DOTS expansion on tuberculosis related outcomes and costs in Haiti. BMC Public Health 2006; 6: 209.

52 Briggs AH. Handling uncertainty in cost-effectiveness models. Pharmacoeconomics 2000; 17: 479-500.

53 Suarez PG, Floyd K, Portocarrero J, et al. Feasibility and cost-effectiveness of standardised second-line drug treatment for chronic tuberculosis patients: a national cohort study in Peru. Lancet 2002; 359: 1980-1989.

54 Murray CJL, Salomon JL. Expanding the WHO tuberculosis control strategy: rethinking the role of active casefinding. Int J Tuberc Lung Dis 1998; 2: S9-S15.

55 Shepard DS, Agness-Soumahoro J, Bail RN, et al. Expenditures on HIV/AIDS: levels and determinants lessons from five countries. AIDS and Development: the role of government, . Limelette: , World Bank, European Union \& UNAIDS, 1996.

56 Floyd K, Hutubessy R, Kliiman K, et al. Cost and cost-effectiveness of multidrug-resistant tuberculosis treatment in Estonia and Russia. Eur Respir J 2012; 40: 133-142.

57 Guo N, Marra F, Marra CA. Measuring health-related quality of life in tuberculosis: a systematic review. Health Qual Life Outcomes 2009; 7: 14.

58 Mallory KF, Churchyard GJ, Kleinschmidt I, et al. The impact of HIV infection on recurrence of tuberculosis in South African gold miners. Int J Tuberc Lung Dis 2000; 4: 455-462.

59 Khan A, Sterling TR, Reves R, et al. Lack of weight gain and relapse risk in a large tuberculosis treatment trial. Am J Respir Crit Care Med 2006; 174: 344-348.

60 Moniruzzaman A, Kazanjian A, Wong H, et al. A systematic review on risk factors of mortality among TB patients. Am J Respir Crit Care Med 2010; 181: A5460.

61 El-Sadr WM, Perlman DC, Denning E, et al. A review of efficacy studies of 6-month short-course therapy for tuberculosis among patients infected with human immunodeficiency virus: differences in study outcomes. Clin Infect Dis 2001; 32: 623-632. 